\title{
Abnormal methylation status of FBXW10 and SMPD3, and associations with clinical characteristics in clear cell renal cell carcinoma
}

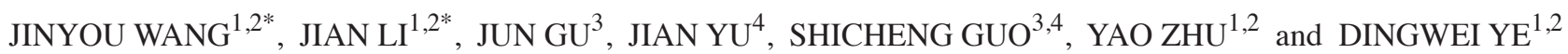 \\ ${ }^{1}$ Department of Oncology, Shanghai Medical College, Fudan University; \\ ${ }^{2}$ Department of Urology, Fudan University Cancer Hospital, Shanghai 200032; \\ ${ }^{3}$ State Key Laboratory of Genetic Engineering and Ministry of Education Key Laboratory of Contemporary Anthropology, \\ School of Life Sciences, Fudan University, Shanghai 200433; ${ }^{4}$ State Key Laboratory of Oncogenes and Related Genes, \\ Shanghai Cancer Institute, Renji Hospital, School of Medicine, Shanghai Jiao Tong University, Shanghai 200240, P.R. China
}

Received March 18,2014; Accepted March 12, 2015

DOI: $10.3892 / \mathrm{ol} .2015 .3707$

\begin{abstract}
The present study aimed to evaluate the use of the $27 \mathrm{~K}$ methylation array to investigate abnormal methylation of two genes and their associations with clinical characteristics in clear cell renal cell carcinoma (ccRCC). Six differentially-methylated genes identified using the $27 \mathrm{~K}$ methylation array were screened in the human RCC 786- 0 cell line and normal kidney tissues by bisulfite sequencing polymerase chain reaction (PCR). Differentially-methylated regions (DMRs) that were abnormally hypermethylated in the cell line were further validated in renal tumor and paired normal tissues by pyrosequencing. The correlations between DMRs and differences (methylation rate of tumor minus that of paired normal tissue) according to gender, age, tumor size, Fuhrman grade and disease stage were assessed. Gene expression prior to and following 5-Aza-2'-deoxycytidine treatment was examined using reverse transcription quantitative PCR (RT-qPCR). Two DMRs located in the FBXW10 and SMPD3 genes were found to be hypermethylated in the 786-0 cells, but not in the normal kidney tissues. Pyrosequencing results showed that the average methylation rate of FBXW10 in the
\end{abstract}

Correspondence to: Professor Dingwei Ye or Dr Yao Zhu, Department of Urology, Fudan University Shanghai Cancer Hospital, 270 Dong'an Road, Shanghai 200032, P.R. China

E-mail: dwyeli@yahoo.com.cn

E-mail: mailzhuyao@gmail.com

*Contributed equally

Abbreviations: ccRCC, clear cell renal cell carcinoma; DMR, differentially-methylated regions; RT-qPCR, reverse transcription quantitative polymerase chain reaction; TCGA, The Cancer Genome Atlas project

Key words: FBXW10, SMPD3, DNA methylation, clear cell renal cell carcinoma cancer tissues was significantly higher compared to that in the paired normal tissues (48.78 vs. $34.62 \%$; $\mathrm{P}<0.001)$. The methylation rate of SMPD3 was also higher in the cancer tissues compared with the paired normal tissues (58.98 vs. $38.66 \%$; $\mathrm{P}<0.001)$. In stage $\mathrm{T} 1 \mathrm{RCC}$, the methylation rate of the tumor tissue was positively correlated with the Fuhrman grade $(\mathrm{P}=0.02)$. The difference in methylation between the tumor and normal tissues was significantly higher in the group with high Fuhrman grade for the two genes. Furthermore, the linear correlation between methylation difference and tumor size was also confirmed $(\mathrm{P}=0.01)$. The RT-qPCR analysis demonstrated that SMPD3 and FBXW10 mRNA expression was significantly upregulated following 5-Aza-2'-deoxycytidine treatment. The results identified two novel DMRs located in SMPD3 and FBXW10 that were hypermethylated in the ccRCC tissue samples. The methylation profile in ccRCC could potentially provide predictive information for clinical decisions.

\section{Introduction}

Renal cell carcinoma (RCC) is one of the most common malignant diseases worldwide, with 330,000 estimated new cases in 2013. Recently, an increased incidence of RCC has been observed in developed and developing countries. Approximately $85 \%$ of RCC is clear cell carcinoma. In the last decade, a significant improvement in the understanding of ccRCC carcinogenesis has resulted in the development of a predictive panel and targeted agents. Epigenetic alteration has been shown to be an important mechanism underlying carcinogenesis $(1,2)$. For example, RASSF1 is a gene associated with RCC that has been previously investigated. Epigenetic alteration has been shown to be a dynamic process (3). In normal tissue, the methylation of genes is different between individuals due to age, exposure and tissue-specific origins (4). Normal tissue may be regarded as the epigenetic background; however, the role of elevated methylation in cancer tissue when compared with the epigenetic background remains unknown. Pyrosequencing is a quantitative method that allows for further elucidation (5). The identification of novel epigenetic mechanisms underlying 
tumorigenesis and epigenetic dynamic processes accompanying tumor progression is warranted.

Furthermore, epigenetic alterations could potentially be of significant clinical value in aiding clinical decisions. The value of epigenetic alteration in predicting Fuhrman grade in T1 stage ccRCC has previously been reported (6); the percentage of global cellular methylation was significantly higher in tumors of grades III and IV (75.93 vs. 93.33\%). As the management of RCC continues to evolve, the methylation status could serve as a useful biomarker that reflects the disease characteristics and biological behaviors.

DNA methylation arrays have recently been developed for the genome-wide profiling of DNA methylation (7). In the current study, candidate differentially-methylated regions (DMRs) were identified based on this high throughput technique and were further screened in the 786-0 cell line derived from primary clear cell adenocarcinoma and in the normal kidney tissues. DMRs that were abnormally hypermethylated in the cell line were further validated in ccRCC tissue and paired normal tissues by pyrosequencing. Abnormal methylation and the associations with clinical characteristics were examined.

\section{Materials and methods}

Methylation microarray based data. DNA methylation profiling data of $418 \mathrm{ccRCC}$ samples, consisting of $219 \mathrm{ccRCC}$ and 199 paired tissues, were obtained from The Cancer Genome Atlas project (TCGA) using the $27 \mathrm{k}$ Illumina Infinium Methylation Beadchip Array (Illumina Inc., San Diego, CA, USA) (8). Using this platform, 27,578 CpG sites in the human genome were assessed and fluorescent signals for methylated (Cy5) and unmethylated (Cy3) alleles provided the methylation level: $\beta=\mathrm{Cy} 5 /(\mathrm{Cy} 3+\mathrm{Cy} 5+100)$, with $\sim 30$ replicate bead measurements per locus. All data were background-corrected and normalized separately according to recommendations for each platform. Subsequent analyses were conducted using $\mathrm{R}$ (a language and environment for statistical computing and graphics) (9). Differentially-hypermethylated CpG sites or genes between groups were identified by fold-change ( $\mathrm{fd}>1.25)$ and t-test $(\mathrm{P}<0.05)$. In the first stage, 2,856 differentially-methylated $\mathrm{CpG}$ sites fulfilled the $\mathrm{P}<0.05$ criterion (the $\mathrm{P}$-value following Bonferroni correction). In the second stage, MBD-Seq data, which have been widely used to identify cancer specific biomarkers $(10,11)$, were used to validate the results of the differentially-methylated genes identified using the $27 \mathrm{~K}$ methylation array, including $5 \mathrm{ccRCC}$ and 5 cancer paired normal tissues.

Cell culture and 5-Aza-2'-deoxycytidine treatment of 786-0 cell line. The RCC 786-0 cell line was purchased from the Type Culture Collection of the Chinese Academy of Sciences (Shanghai, China) and routinely maintained in Dulbecco's modified Eagle's medium (DMEM; Invitrogen, San Diego, CA, USA) supplemented with $10 \%$ fetal calf serum (FCS) at $37^{\circ} \mathrm{C}$ in $5 \% \mathrm{CO}_{2}$. Total DNA and RNA were isolated. The demethylating agent, 5-Aza-2'-deoxycytidine (Sigma-Aldrich, Dorset, UK), was freshly prepared in $\mathrm{ddH}_{2} \mathrm{O}$ and filter sterilized. The cell line was plated in $75-\mathrm{cm}^{2}$ flasks in DMEM supplemented with $10 \%$ FCS at differing densities, depending on their doubling time, to ensure that control and 5-Aza-2'-deoxycytidine-treated lines reached 75\% confluency at the point of RNA extraction. After $24 \mathrm{~h}$, the cells were treated with 5 mM 5-Aza-2'-deoxycytidine. The medium was changed $24 \mathrm{~h}$ after treatment and then changed again after 72 h. Following 5-Aza-2'-deoxycytidine treatment, total RNA was isolated from the cell line. The DNA and RNA of the cell line and tissues were isolated using the DNA/RNA Isolation kit (Tiangen Biotech Co., Lt., Beijing, China), according to the manufacturer's instructions.

Tissue samples. A total of 85 paired tissue samples from patients who underwent surgery for newly diagnosed ccRCC in Fudan University Shanghai Cancer Center (Shanghai, China) were investigated. Each case consisted of a sample of cancer tissue and matched paired macroscopically normal renal tissue (distant from the tumor). The tissues were stored at $-80^{\circ} \mathrm{C}$ in the tissue bank. Additionally, one case diagnosed with benign kidney angiomyolipoma was examined. Written informed consent was obtained from the patients and the study was approved by the Medical Institutional Review Board of Fudan University Shanghai Cancer Center.

Bisulfite modification and methylation analysis, and bisulfite sequencing. For bisulfite conversion, the EZ DNA Methylation-Gold ${ }^{\mathrm{TM}}$ kit from Zymo Research Corporation (Irvine, CA, USA) was used for optimized bisulfite conversion; $500 \mathrm{ng}$ of genomic DNA and the manufacturer's instructions were utilized. DNA was denatured by the addition of Zymo M-Dilution buffer (containing $\mathrm{NaOH}$ : Zymo Research Corporation) and incubated at $98^{\circ} \mathrm{C}$ for $10 \mathrm{~min}$, followed by $64^{\circ} \mathrm{C}$ for $2.5 \mathrm{~h}$ in a thermocycler. The methylation-specific primers were designed based on the sequencing data of the PCR-amplified bisulfite-modified cell line genome using MethPrimer software (http://www. urogene.org/methprimer/). The DNA primers are listed in Table I. PCR was performed under the following conditions: Denaturation at $95^{\circ} \mathrm{C}$ for $5 \mathrm{~min}$, followed by 42 cycles of $95^{\circ} \mathrm{C}$ for $30 \mathrm{sec}, 57^{\circ} \mathrm{C}$ for $30 \mathrm{sec}$ and $72^{\circ} \mathrm{C}$ for $40 \mathrm{sec}$ with a final extension step at $72^{\circ} \mathrm{C}$ for $10 \mathrm{~min}$. The PCR amplified fragments were cloned into pMD19-T (Takara Bio, Inc., Otsu, Shiga, Japan) for $2 \mathrm{~h}$, followed by transformation into DH5 $\alpha$. A total of 8 clones were selected for PCR validation. Transformants containing recombinant plasmids were selected by blue/white colony screening and sequencing, as previously described (12). The bisulfite sequencing PCR (BSP) methylation percentage was calculated as the number of methylated cytosines divided by the total number of cytosines in all of the amplicons analyzed.

Pyrosequencing. To demonstrate the relative degree of methylation, DNA isolated from RCC and matched normal tissues were analyzed using pyrosequencing. Measurements were performed, followed by pyrosequencing with PyroMark Gold Q96 Reagents, using the PyroMark Q24 pyrosequencing system (Qiagen GmbH, Hilden, Germany), according to the manufacturer's instructions. The sequencing assay was validated using an internal control (a non-CpG cytosine within the target methylation sequence region). The primers are listed in Table I. 
Table I. Primers.

\begin{tabular}{|c|c|}
\hline Gene & Sequence $\left(5^{\prime}-3^{\prime}\right)$ \\
\hline \multicolumn{2}{|l|}{ BSP } \\
\hline \multicolumn{2}{|c|}{ FBXW10 } \\
\hline $\mathrm{F}$ & TTATTTATTTTTGTTTTGGGAG \\
\hline $\mathrm{R}$ & TTCAAAATACCAACTAAAAACC \\
\hline \multicolumn{2}{|c|}{ SMPD3 } \\
\hline $\mathrm{F}$ & TTTTTTGGTTTTAGGGTTTTGT \\
\hline $\mathrm{R}$ & TCTCRACTTAAAACCCCAA \\
\hline \multicolumn{2}{|l|}{ CD9 } \\
\hline $\mathrm{F}$ & TTTTTATAAGTGAYGTTGGGGG \\
\hline $\mathrm{R}$ & CTAAACAATCCCCAAACRCTT \\
\hline \multicolumn{2}{|c|}{ HIST1H3E } \\
\hline $\mathrm{F}$ & TTGAAAAAATAAATTAATYGTGAA \\
\hline $\mathrm{R}$ & AAATCCTAAACTATTTCTCRCA \\
\hline \multicolumn{2}{|l|}{ LEP } \\
\hline $\mathrm{F}$ & GGAGTTGAGGATGGAGATTTAT \\
\hline $\mathrm{R}$ & AACTCCRACRCRACTATAA \\
\hline \multicolumn{2}{|c|}{ GGT6 } \\
\hline $\mathrm{F}$ & AGGAGATATTAGAGGYGTTGGT \\
\hline $\mathrm{R}$ & ACAAACCCAACCAAACRTAAT \\
\hline \multicolumn{2}{|c|}{ Pyrosequencing } \\
\hline \multicolumn{2}{|c|}{ FBXW10 } \\
\hline $\mathrm{F}$ & $\begin{array}{l}\text { TTGGGAGAAGTTTGTAATAGAAAA } \\
\text { GGTA }\end{array}$ \\
\hline $\mathrm{R}$ & $\begin{array}{l}\text { Biotin-CCAAATTTTCCATAATCCTAA } \\
\text { AATAACC }\end{array}$ \\
\hline Seq & AAGGTATAATTGGGG \\
\hline \multicolumn{2}{|c|}{ SMPD3 } \\
\hline $\mathrm{F}$ & GGGTGGAGGAAAGTATTGATAT \\
\hline $\mathrm{R}$ & $\begin{array}{l}\text { Biotin-CTATCCTCCTACATCCCCCTA } \\
\text { CACTAC }\end{array}$ \\
\hline Seq & TGGAGGAAAGTATTGATATT \\
\hline \multicolumn{2}{|c|}{ RT-qPCR } \\
\hline \multicolumn{2}{|c|}{ FBXW10 } \\
\hline $\mathrm{F}$ & TCCTCCTGACTGTTAGCG \\
\hline $\mathrm{R}$ & AACTGCACGTTGGATTGA \\
\hline \multicolumn{2}{|c|}{ SMPD3 } \\
\hline $\mathrm{F}$ & СCTTTGCGTTTCTCGGCTTTC \\
\hline $\mathrm{R}$ & CCCGTGCCCTTCCATTCA \\
\hline
\end{tabular}

RT-qPCR, reverse transcription quantitative polymerase chain reaction; F, forward; R, reverse; Seq, sequencing primer 1.

$R T$ - $q P C R$. Total RNA $(1 \mu \mathrm{g})$ was used for cDNA synthesis using the First Strand cDNA Synthesis kit (Fermentas, Pittsburgh, PA, USA) following the manufacturer's protocol. RT-qPCR was performed on PRISM 7500, (Applied Biosystems Life Technologies, Foster City, CA, US) using SYBR Premix Ex Tag (Takara Bio Inc.). PCR was performed under the following conditions: Denaturation at $95^{\circ} \mathrm{C}$ for $10 \mathrm{~min}$, followed by 40 cycles of $95^{\circ} \mathrm{C}$ for $10 \mathrm{sec}$ and $57^{\circ} \mathrm{C}$ for $20 \mathrm{sec}$ with a final extension step at $72^{\circ} \mathrm{C}$ for $15 \mathrm{sec}$. Quantification of mRNA expression was performed using the $\Delta$ ct method ( $\Delta$ ct sample - $\Delta$ ct calibrator). The primers are listed in Table I.

Statistical analysis. Methylation rate and the difference between RCC and paired normal tissues (methylation rate of RCC minus that of paired normal tissues) were analyzed.

A paired Student's t-test was used to examine differences between the cancer and matched normal tissues. Univariate analysis of variance or liner correlation was used to evaluate the association between methylation status and gender, age, Fuhrman grade, disease stage and metastasis. $\mathrm{P}<0.05$ was used to indicate a statistically significant difference in all tests.

\section{Results}

Methylation $27 \mathrm{~K}$ microarray data results and locations of DMRs. At least 10 differentially-hypermethylated $\mathrm{CpG}$ sites that belonged to 6 differentially-hypermethylated regions were identified. The 6 DMRs were located in 6 genes. After searching Pubmed, four DMRs were identified in the promoter or the first exon of LEP, HIST1H3E, CD9 and FBXW10. Another 2 identified DMRs were located in $\mathrm{CpG}$ islands belonging to the first intron of SMPD3 and GGT6, respectively (Table II).

Methylation status of DMRs in the RCC 786-0 cell line and normal tissues. First, the candidate DMR regions were validated in the RCC 786-0 cell line and normal renal tissues using BSP. Two DMRs located in SMPD3 and FBXW10 were found to be hypermethylated in the 786-0 cells only. The methylation rate of FBXW10 was $84.4 \%$ in the $786-0$ cells versus $22.2 \%$ in the normal tissues (Fig. 1A), while the methylation rate of SMPD3 was $82.9 \%$ in the $786-0$ cells versus $35.7 \%$ in the normal tissues (Fig. 1B). Additionally, 4 other DMRs showed hypermethylation in 786-0 cells and normal tissues.

Validation of FBXW10 and SMPD3 in 85 paired $R C C$ tissues. Further validation of the 2 hypermethylated DMRs was performed by pyrosequencing in 85 paired RCC tissues. Pyrosequencing of FBXW10 included $4 \mathrm{CpG}$ sites (the 2nd to 5th $\mathrm{CpG}$ sites in BSP), and SMPD3 included $7 \mathrm{CpG}$ sites (the 6th to 12 th $\mathrm{CpG}$ sites in BSP) (Fig. 1A and B). The average methylation rate of FBXW10 in the cancer tissues was significantly higher compared with the paired normal tissues (48.78 vs. $34.62 \%$; $\mathrm{P}<0.001$; Fig. $1 \mathrm{C}$ ). Moreover, the methylation rate of SMPD3 was also higher in the cancer tissues compared with the paired normal tissues (58.98 vs. $38.66 \%$; $\mathrm{P}<0.001$; Fig. 1D).

Methylation of FBXW10 and SMPD3 and their associations with clinical characteristics in ccRCC. A total of $90.59 \%$ (77/85) cases were hypermethylated in the RCC tissues. Upon univariate analysis, FBXW10 methylation did not correlate with gender, age, tumor size, Fuhrman grade or disease stage. In stage $\mathrm{T} 1$ disease, the methylation rate of the RCC tissues was positively correlated with Fuhrman grade ( $\mathrm{P}=0.020$; Fig. 2A). The methylation rate of FBXW10 in Fuhrman grades I, II and III was $51.89,60.37$ and $66.18 \%$, respectively. Notably, the 
A

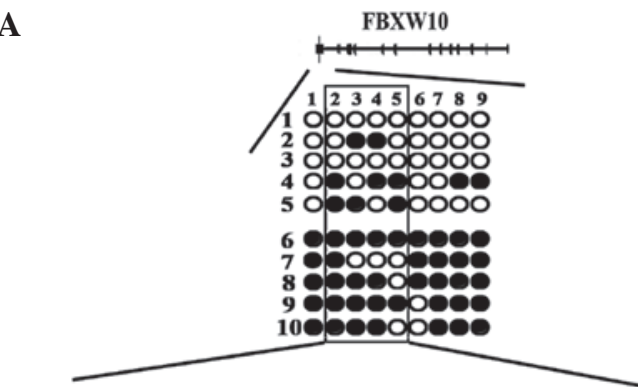

B3: TATTTATTTATTTTTTYYGTTTTTTTAGTGTTTGGTGGYGTGTYGTTGTAAGTGYTA

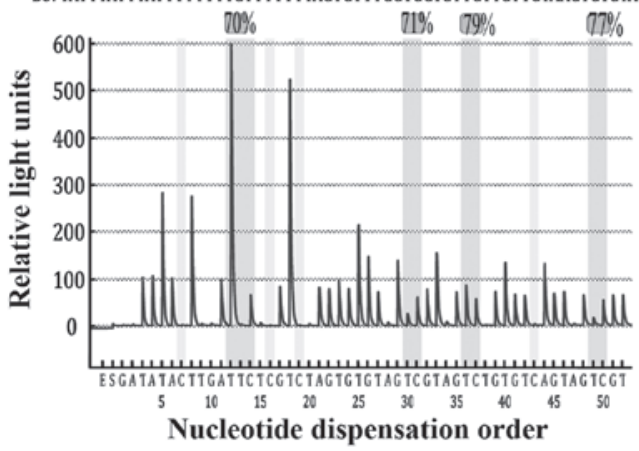

C

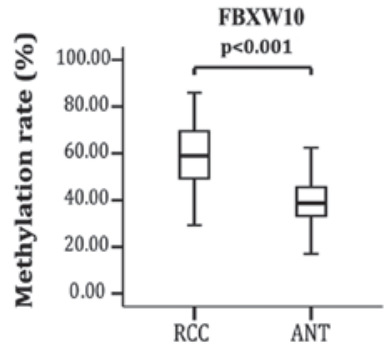

Tissue type

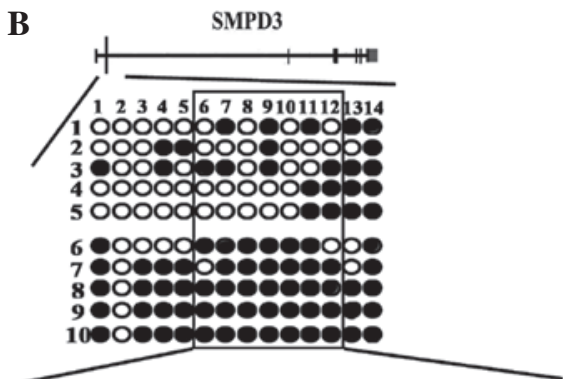

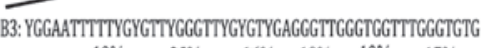

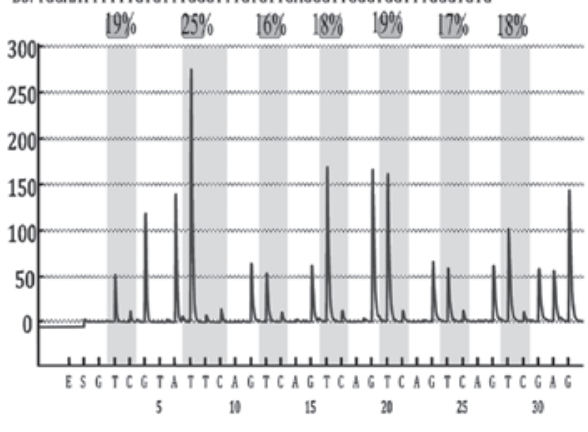

D

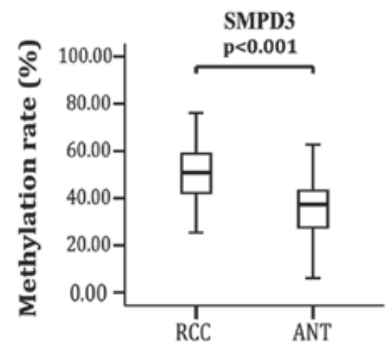

Tissue type

Figure 1. Gene location and bisulfite sequencing polymerase chain reaction (BSP) and pyrosequencing results: (A) Gene location, BSP and representative result of pyrosequencing in FBXW10; (B) gene location, BSP and representative result of prosequencing in SMPD3; (C and D) methylation level of two genes in tumor and paired normal tissues. RCC, renal cell carcinoma; ANT, adjacent normal tissue.

A

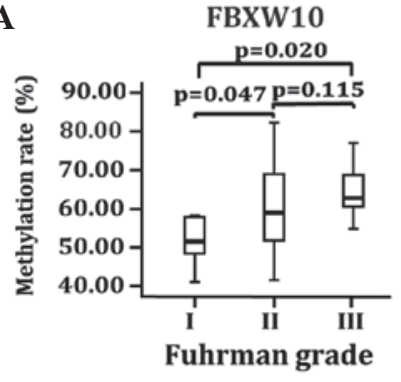

D

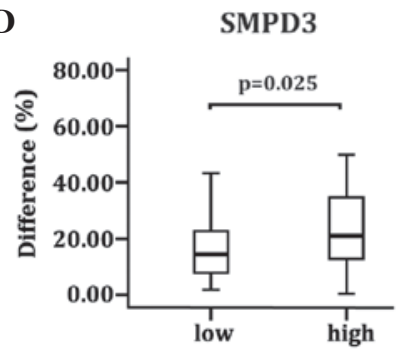

Fuhrman grade
B

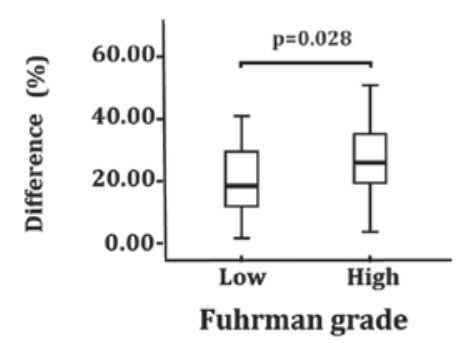

$\mathbf{E}$

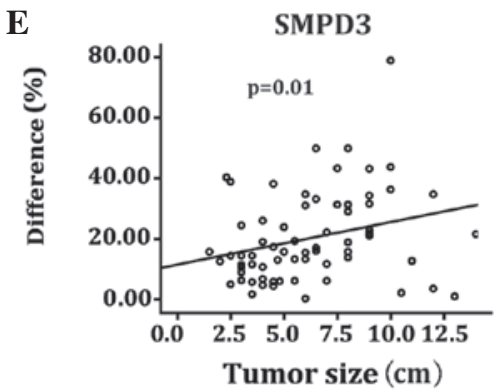

C
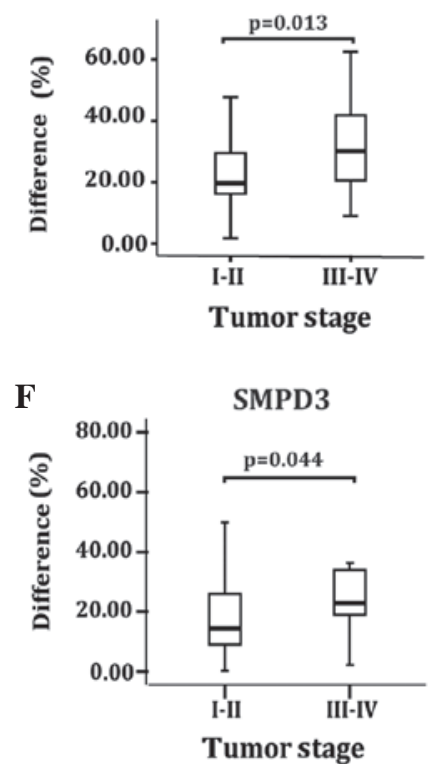

Figure 2. Methylation of two genes and their associations with clinical characteristics in ccRCC. (A) Correlation of FBXW10 methylation level with Fuhrman grade in T1 stage ccRCC. (B) Correlation of methylation difference in FBXW10 with Fuhrman grade group. (C) Correlation of methylation difference in FBXW10 with tumor stage. (D) Correlation of methylation difference in SMPD3 with Fuhrman grade group. (E) Linear correlation of methylation difference in SMPD3 with tumor size. (F) Correlation of methylation difference in SMPD3 with tumor stage. ccRCC, clear cell renal cell carcinoma. 
Table II. Genes showing significant differential methylation between the clear cell renall cell carcinoma and paired adjacent tissues

\begin{tabular}{|c|c|c|c|c|c|c|}
\hline CpG name & Gene name & P-value & P-value FDR & P-value bonferroni & Fold-change & Location \\
\hline cg05127924 & FBXW10 & $2.11 \times 10^{-73}$ & $5.28 \times 10^{-71}$ & $5.28 \times 10^{-69}$ & 1.914547176 & Exon 1 \\
\hline cg00891541 & SMPD3 & $5.39 \times 10^{-70}$ & $1.07 \times 10^{-67}$ & $1.35 \times 10^{-65}$ & 1.899386384 & Intron 1 \\
\hline $\operatorname{cg} 10556064$ & SMPD3 & $2.65 \times 10^{-57}$ & $2.73 \times 10^{-55}$ & $6.63 \times 10^{-53}$ & 1.682782468 & Intron 1 \\
\hline cg19297232 & SMPD3 & $5.06 \times 10^{-20}$ & $4.78 \times 10^{-19}$ & $1.26 \times 10^{-15}$ & 1.467510342 & Intron 1 \\
\hline $\operatorname{cg} 04511534$ & GGT6 & $3.46 \times 10^{-110}$ & $7.21 \times 10^{-107}$ & $8.65 \times 10^{-106}$ & 1.891379745 & Intron 1 \\
\hline $\operatorname{cg} 22628873$ & GGT6 & $1.16 \times 10^{-42}$ & $4.58 \times 10^{-41}$ & $2.89 \times 10^{-38}$ & 1.649480657 & Intron 1 \\
\hline cg08519905 & CD9 & $7.57 \times 10^{-63}$ & $1.06 \times 10^{-60}$ & $1.89 \times 10^{-58}$ & 1.906118383 & Promoter \\
\hline cg07922606 & HIST1H3E & $2.69 \times 10^{-64}$ & $4.15 \times 10^{-62}$ & $6.72 \times 10^{-60}$ & 2.009304856 & Promoter \\
\hline $\operatorname{cg} 12782180$ & LEP & $1.06 \times 10^{-73}$ & $2.69 \times 10^{-71}$ & $2.64 \times 10^{-69}$ & 2.208969818 & Promoter \\
\hline $\operatorname{cg} 19594666$ & LEP & $2.42 \times 10^{-22}$ & $2.63 \times 10^{-21}$ & $6.05 \times 10^{-18}$ & 1.550117434 & Promoter \\
\hline
\end{tabular}

FDR, false discovery rate.

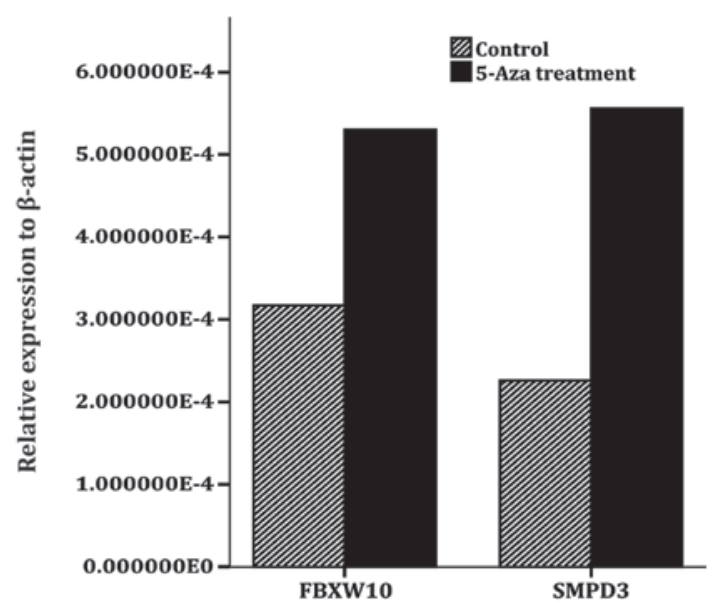

Figure 3. Expression of two genes prior to and following treatment with 5-Aza-2'-deoxycytidine (5'Aza).

difference in methylation between the RCC and paired normal tissues was higher in the high Fuhrman grades (Fuhrman grade III-IV) compared with the low Fuhrman grades (Fuhrman grade I-II). The difference in methylation rate between the high and low Fuhrman grades was 27.24 and $21.02 \%$, respectively $(\mathrm{P}=0.028$; Table III, Fig. 2B). Furthermore, the difference in high stage RCC (III-IV; 31.94\%) was greater than that in low stage RCC (I-II; 22.35\%) ( $\mathrm{P}=0.013$; Table III; Fig. 2C).

A total of $80 \%(68 / 85)$ of cases were hypermethylated in the RCC tissues. There were no significant correlations between SMPD3 methylation rate and gender, age, tumor size, Fuhrman grade and disease stage in the RCC tissues. Significant methylation differences between tumor and normal tissues were found between Fuhrman grade groups. The methylation difference was higher in the high Fuhrman grade groups (Fuhrman grade III-IV) compared with the low Fuhrman grade groups (Fuhrman grade I-II) $(\mathrm{P}=0.025)$. The difference in methylation rate in the high and low Fuhrman grade groups was 24.29 and $16.33 \%$, respectively (Table III; Fig. 2D). The difference was $28.38 \%$ in the high tumor stage (III-IV) group and $18.62 \%$ in the low tumor stage (I-II) group, which was statistically significant
$(\mathrm{P}=0.044)$ (Table III; Fig. 2E). There was a linear correlation between the difference in methylation rate and the tumor size; the larger the tumor, the greater the difference $(\mathrm{P}=0.01)$ (Table III; Fig. 2F).

Gene expression. The RT-qPCR analysis demonstrated that SMPD3 and FBXW10 mRNA expression was upregulated following 5-Aza-2'-deoxycytidine treatment in the RCC cell line. The expression of SMPD3 was increased 2.47 times and that of FBXW10 was increased 1.67 times (Fig. 3).

\section{Discussion}

The Illumina $27 \mathrm{k}$ methylation array platform is one of the most comprehensive microarrays currently available for genome-wide DNA methylation analysis. The array integrates 27,578 CpG sites (CpGs) at single-nucleotide resolution, covering $>14,000$ RefSeq genes (13). The $27 \mathrm{~K}$ methylation data from TCGA contains 418 ccRCC-associated samples. TCGA supplies a collection of clinical and genomic data in which associations between clinical value and genetics can be investigated (14). In the present study, 2,856 differentially-methylated $\mathrm{CpG}$ sites were identified, and then five ccRCC and five normal tissue paired samples were used to validate and confirm two DMRs.

FBXW10 is located in 17p12. F-box protein family members, such as FBXW10, are characterized by an F-box motif of $\sim 40$-amino acid and act as protein-ubiquitin ligases (15). FBXW7, a member of this family that has been widely investigated, is believed to act as a tumor suppressor by negative regulation of a number of oncogenic proteins (16). The function of FBXW10 has not been studied as well as methylation profiling. To the best of our knowledge, this is the first study to report the abnormal hypermethylation of FBXW10 in RCC. SMPD3, also known as NSMase2, is located in 16q22.1 and is a member of the neutral sphingomyelinase family, the major function of which is to catalyze the hydrolysis of sphingomyelin in biological membranes to ceramide and phosphorylcholine. Among the enzymes, SMPD3 has been the most widely studied tumor suppressor. SMPD3 has also been implicated in cell growth inhibition and tumorigenesis (17). 
Table III. Clinicopathological features of hypermethylated cases.

\begin{tabular}{|c|c|c|c|c|c|}
\hline Feature & Value & $\%^{\mathrm{b}}$ & P-value & $\%^{\mathrm{c}}$ & P-value \\
\hline \multicolumn{6}{|l|}{ FBXW10 } \\
\hline Gender $^{\mathrm{a}}$ & & & 0.051 & & 0.053 \\
\hline Male & $48(62.34)$ & 63.39 & & 25.97 & \\
\hline Female & $29(37.66)$ & 57.96 & & 20.34 & \\
\hline Furhman grade $^{a}$ & & & 0.162 & & 0.028 \\
\hline I, II & $42(54.55)$ & 59.61 & & 21.02 & \\
\hline III, IV & $35(45.45)$ & 63.42 & & 27.24 & \\
\hline Tumor stage $^{\mathrm{a}}$ & & & 0.069 & & 0.013 \\
\hline I, II & $65(84.42)$ & 60.28 & & 22.35 & \\
\hline III, IV & $12(15.58)$ & 67.06 & & 31.94 & \\
\hline Mean age (range), years & $55(30-84)$ & & 0.388 & & 0.344 \\
\hline Mean tumor size (range), $\mathrm{cm}$ & $6(1.5-14)$ & & 0.779 & & 0.402 \\
\hline \multicolumn{6}{|l|}{ SMPD3 } \\
\hline Gender $^{\mathrm{a}}$ & & & 0.113 & & 0.786 \\
\hline Male & $43(63.24)$ & 54.29 & & 20.56 & \\
\hline Female & $25(36.76)$ & 48.55 & & 19.55 & \\
\hline Furhman grade $^{\mathrm{a}}$ & & & 0.984 & & 0.025 \\
\hline I, II & $35(51.47)$ & 52.21 & & 16.33 & \\
\hline III, IV & $33(48.53)$ & 52.14 & & 24.29 & \\
\hline Tumor stage $^{a}$ & & & 0.419 & & 0.044 \\
\hline I, II & $57(83.82)$ & 51.55 & & 18.62 & \\
\hline III, IV & $11(16.18)$ & 55.42 & & 28.38 & \\
\hline Mean age (range), years & $55(30-84)$ & & 0.202 & & 0.271 \\
\hline Mean tumor size (range), $\mathrm{cm}$ & $6(1.5-14)$ & & 0.123 & & 0.010 \\
\hline
\end{tabular}

${ }^{\mathrm{a}}$ Data presented as cases, $\mathrm{n}(\%)$; ${ }^{\mathrm{R} C C}$ methylation rate; ${ }^{\mathrm{c}}$ difference in methylation rate between $\mathrm{RCC}$ and paired normal tissues.

A

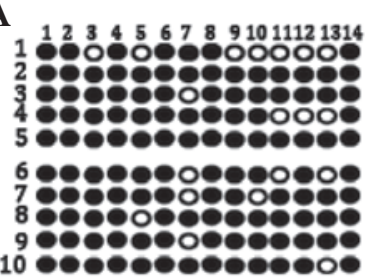

B

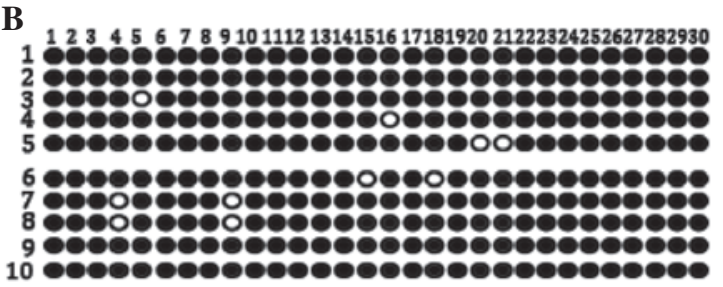

10 90909
C

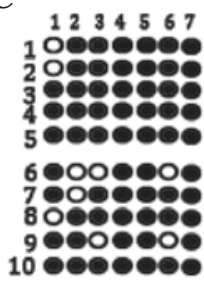

D

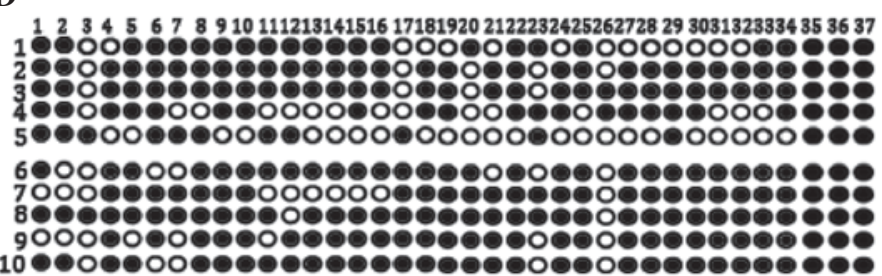

Figure 4. Bisulfite sequencing polymerase chain reaction results for 4 other genes: (A) CD9, (B) HIST1H3E, (C) GGT6 and (D) LEP.

The promoter of SMPD3 was identified by hypermethylation in breast and colorectal cancer cells $(18,19)$. The identification was based on high throughput screening; however, the methylation status in ccRCC has not been reported to date.
The DMR of FBXW10 is located in the head of the first exon. Hypermethylation has been previously demonstrated in cell lines and tumor tissues (20). As the first exon is a common locus that causes epigenetic inactivation, the present study 
analyzed further gene expression regulated by methylation using demethylation treatment. The upregulation of expression following 5-Aza-2'-deoxycytidine treatment indicated that FBXW10 exhibited epigenetic inactivation by hypermethylation of the first exon. The DMR of SMPD3 was located in the head of the first intron within $1 \mathrm{~kb}$ downstream of the first exon. As a tumor suppressor that has been widely studied, the promoter of SMPD3 was identified by hypermethylation in breast and colorectal cancer cells (17). In the present study, a novel hypermethylated DMR was identified in the ccRCC cells and tissues. Although it is not a traditional region of inactivated gene expression, further demethylation treatment caused upregulation of expression. Previous studies have shown that the hypermethylation of introns also regulates gene expression $(21,22)$. The underlying mechanisms may involve binding proteins that regulate methylation and methylation of the first intron of SMPD3, thus affecting gene expression (21). In the present study, it was reported for the first time that novel hypermethylation is another potential mechanism that downregulates gene expression, with the exception of promoter methylation in SMPD3.

The methylation rate of FBXW10 correlated with the Fuhrman grade in T1 stage ccRCC. The higher the methylation rate, the higher the Fuhrman grade. Due to the unsatisfactory accuracy of the Fuhrman grade and percutaneous renal biopsy, the methylation rate of FBXW10 could serve as a beneficial biomarker to improve the accuracy of diagnosis. T1 stage ccRCC with FBXW10 hypermethylation tends to be highly invasive. Thus, this additional tool could aid in the identification of the optimal treatment of T1 stage ccRCC (23-25). A previous study showed that FBXW10 acted as a protein-ubiquitin ligase. F-box proteins interact with SKP1 via F box, and interact with ubiquitination targets through other protein interaction domains (15). The present study is the first to identify methylation profiling as a biomarker; it was demonstrated that FBXW10 was inactivated by methylation of the first exon, and that inactivation of FBXW10 may be significant in the formation of a highly invasive tumor. The paired normal tissue chosen in this study was normal renal cortex tissue distant from the tumor. As the methylation of genes is different in individuals due to age, exposure and tissue-specific origins, the methylation status of the paired normal tissue could be regarded as the epigenetic background of the patient. In the present study, the difference was defined as the methylation rate of $\mathrm{RCC}$ minus the methylation rate of the paired normal tissue. This enabled investigation of the dynamic process of DNA methylation. The results indicated that not only the RCC methylation rate was important, but that it also reflected the difference between participants with high Fuhrman grade formation and progression of local tumor. This is the first study to demonstrate the link between cancer and individualized epigenetic background. However, the function of FBXW10 in ccRCC requires further elucidation.

The correlation of the SMPD3 methylation rate with clinical features also exhibited differences. Moreover, the results indicated differences in the participants with high Fuhrman grade formation and tumor progression. Linear correlation analysis indicated that the larger the tumor, the higher the methylation rate in RCC compared to the background. This dynamic process contributes to tumor growth.
The present study was not without limitations. All tumor stages were selected to obtain a general sense of methylation profiling. Larger samples could be studied to confirm the value of FBXW10 as a biomarker. Further studies could reveal the function of methylation regulation of the two novel genes.

In conclusion, two novel DMRs located in SMPD3 and FBXW10 were found to be hypermethylated in ccRCC tissues, and participants exhibited hypermethylated inactivation and tumor genes. FBXW10 was a useful biomarker to predict Fuhrman grade in T1 stage tumors and improved the accuracy of percutaneous renal biopsy. The findings suggest that it is not the methylation rate of $\mathrm{RCC}$, but rather the dynamic methylation process that is important in participants with high Fuhrman grade formation and tumor stage.

\section{References}

1. Morrissey C, Martinez A, Zatyka M, et al: Epigenetic inactivation of the RASSF1A 3p21.3 tumor suppressor gene in both clear cell and papillary renal cell carcinoma. Cancer Res 61: 7277-7281, 2001.

2. Kawai Y, Sakano S, Suehiro Y, et al: Methylation level of the RASSF1A promoter is an independent prognostic factor for clear-cell renal cell carcinoma. Ann Oncol 21: 1612-1617, 2010.

3. Baylin SB and Jones PA: A decade of exploring the cancer epigenome-biological and translational implications. Nat Rev Cancer 11: 726-734, 2011.

4. Christensen BC, Houseman EA, Marsit CJ, et al: Aging and environmental exposures alter tissue-specific DNA methylation dependent upon CpG island context. PLoS Genet 5: e1000602, 2009.

5. Gharizadeh B, Nordström T, Ahmadian A, et al: Long-read pyrosequencing using pure 2'-deoxyadenosine-5'-O'-(1-thiotriphosphate) Sp-isomer. Anal Biochem 301: 82-90, 2002.

6. Minardi D, Lucarini G, Filosa A, et al: Prognostic role of global DNA-methylation and histone acetylation in pT1a clear cell renal carcinoma in partial nephrectomy specimens. J Cell Mol Med 13: 2115-2121, 2009.

7. Houseman EA, Accomando WP, Koestler DC, et al: DNA methylation arrays as surrogate measures of cell mixture distribution. BMC Bioinformatics 13: 86, 2012.

8. Bibikova M, Le J, Barnes B, et al: Genome-wide DNA methylation profiling using Infinium ${ }^{\circledR}$ assay. Epigenomics 1: 177-200, 2009.

9. Dessau RB and Pipper CB: 'R'-project for statistical computing. Ugeskr Laeger 170: 328-330, 2008 (In Danish).

10. Zhao Y, Guo S, Sun J, et al: Methylcap-seq reveals novel DNA methylation markers for the diagnosis and recurrence prediction of bladder cancer in a Chinese population. PLoS One 7: e35175, 2012.

11. He Y, Cui Y, Wang W, et al: Hypomethylation of the hsa-miR-191 locus causes high expression of hsa-mir-191 and promotes the epithelial-to-mesenchymal transition in hepatocellular carcinoma. Neoplasia 13: 841-853, 2011.

12. Yu J, Zhu T, Wang Z, et al: A novel set of DNA methylation markers in urine sediments for sensitive/specific detection of bladder cancer. Clin Cancer Res 13: 7296-7304, 2007.

13. Chen YA, Choufani S, Ferreira JC, Grafodatskaya D, ButcherDT and Weksberg R: Sequence overlap between autosomal and sex-linked probes on the Illumina HumanMethylation 27 microarray. Genomics 97: 214-222, 2011.

14. Braun R, Finney R, Yan C, et al: Discovery analysis of TCGA data reveals association between germline genotype and survival in ovarian cancer patients. PLoS One 8: e55037, 2013.

15. Jin J, Cardozo T, Lovering RC, Elledge SJ, Pagano M and Harper JW: Systematic analysis and nomenclature of mammalian F-box proteins. Genes Dev 18: 2573-2580, 2004.

16. Zhou ZY, Tu KS, Zhang J, et al: Expression of Fbxw7 and its correlation with cell proliferation in human hepatocellular carcinoma. Xi Bao Yu Fen Zi Mian Yi Xue Za Zhi 28: 1303-1306, 2012 (In Chinese).

17. Clarke CJ, Guthrie JM and Hannun YA: Regulation of neutral sphingomyelinase-2 (nSMase2) by tumor necrosis factor-alpha involves protein kinase C-delta in lung epithelial cells. Mol Pharmacol 74: 1022-1032, 2008. 
18. Demircan B, Dyer LM, Gerace M, Lobenhofer EK, Robertson KD and Brown KD: Comparative epigenomics of human and mouse mammary tumors. Genes Chromosomes Cancer 48: 83-97, 2009.

19. Shen Y, Takahashi M, Byun HM, et al: Boswellic acid induces epigenetic alterations by modulating DNA methylation in colorecta cancer cells. Cancer Biol Ther 13: 542-552, 2012.

20. Chaturvedi P and Parnaik VK: Lamin A rod domain mutants target heterochromatin protein 1alpha and beta for proteasomal degradation by activation of F-box protein, FBXW10. PLoS One 5: e10620, 2010

21. Tang Y, Liu C, Wang X, Liu D, Ingvarsson S and Chen $\mathrm{H}$ : Demethylation of the region around exon 2 of MLH1 gene in gastrointestinal cancer. Anticancer Res 32: 4861-4864, 2012.
22. Godler DE, Slater HR, Bui QM, et al: FMR1 intron 1 methylation predicts FMRP expression in blood of female carriers of expanded FMR1 alleles. J Mol Diagn 13: 528-536, 2011.

23. Smaldone MC and Uzzo RG: Active surveillance: a potential strategy for select patients with small renal masses. Future Oncol 7: 1133-1147, 2011.

24. Lane BR, Tobert CM and Riedinger CB: Growth kinetics and active surveillance for small renal masses. Curr Opin Urol 22: 353-359, 2012

25. Mason RJ, Abdolell M, Trottier G, et al: Growth kinetics of renal masses: analysis of a prospective cohort of patients undergoing active surveillance. Eur Urol 59: 863-867, 2011. 